center or axis of the canal, and overcome completely by a force exerted along that axis, as illustrated at the superior strait; but when the resistances are unequal and the force is applied in the axis of the canal, the body is arrested at the point of greatest resistance, and the force continuing, it is propelled in the direction of the lesser resistance, as well as in that of the axis of the canal. In fact, it rotates around that point of greater resistance, as around a pivot, thus producing extension of the head, and increasing the antero-posterior diameter of the presenting part.

If the two unequal and vertical resistant forces $b$ a $d c$, (Fig. 7) be applied to the body, $a c$, they can be replaced by a single vertical force, not passing through the center $o$ of the body $a c$, but through a point corresponding to its percussion point, approaching the greater resistance, $d c$, in proportion to the greater weakness of the resistance, $b a$. If, for example, the resistances were in the proportion of 1 to 3 , these resistances would be equally overcome if the force were applied three times nearer the point of greater resistance. But, since this is impracticable, and an axial force, of tends to propel the body, $a c$, backward toward $a$ and downward toward $f$, or along their any resultant $0, e$, in an oblique direction downward and backward; to relieve the strain on the

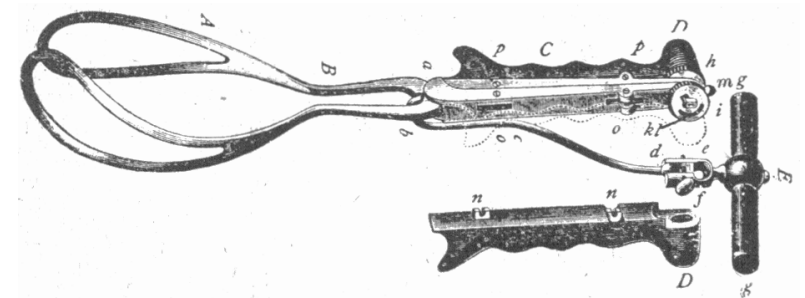

Fig. 8.-A, blade; H, shanks: C, handle; DD, detachable rubber side pieces; E, the axis traction attachment: a, socket between shank: for book $b$, of traction rod; $d$, bull end of rod; $e$ clamp socket for $d ; f$, thumb-screw for screwing the ball and socket joint; $g$, the rubber cross whe i, the ordinary Elliot wheel and threaled bar; i, an additional Wheel with a hali-threated slot on opposite side, which both form the wheel or small knob on lock-wheel; $\mathrm{n}, \mathrm{n}$, metallic lugs which hold side pieces in place; oo, grooves in the metal for the reception of the lugs pp. screws passing antero posteriorly through the metal and flat where unlocks the side pieces securely to the metallic portion.

weaker structure, the perineum, we have only to divert the oblique direction of the force $o e$ into the opposite oblique direction, $o d$. Thus it will be observed that this anterior oblique direction of the force, $o d$, corresponds with the oblique direction of the force $f p$ (Fig. 5) in the ordinary curved forceps, as demonstrated above.

Hence, the proper or perfect obstetric forceps is one in which the force can be expressed in the axis of the superior strait, and in the anterior oblique direction at the outlet.

The forceps which I have the pleasure of exhibiting to you, (Fig. 8) consist of the old familiar Simp. son blades and shanks, the Elliott handles, and this simple traction rod, and an exceedingly practical lock, and permits admirably of just such expression of the force (Fig. 9). At the superior strait it is an excellent axis-traction forceps; at the outlet it is the most desirable model of the ordinary forceps. Though the fetal head is free to rotate, the instrument can not slip. It is not a Tarnier model, and possesses none of the defects of such; nor does it require an expert to use it. Whoever can use an ordinary forceps can use this as readily and as successfully as any expert. It does not produce that feeling of inse- curity and uncertainty, for the muscular sense of the operator is always called into play the same as with the common forceps.

The perineum may be pushed back by the shanks. of the instrument, without seriously altering the position of the blades, just as in the use of the common forceps. This, in some cases, is an absolute necessity, depending upon the situation of the posterior commissure of the vulva. This adaptability

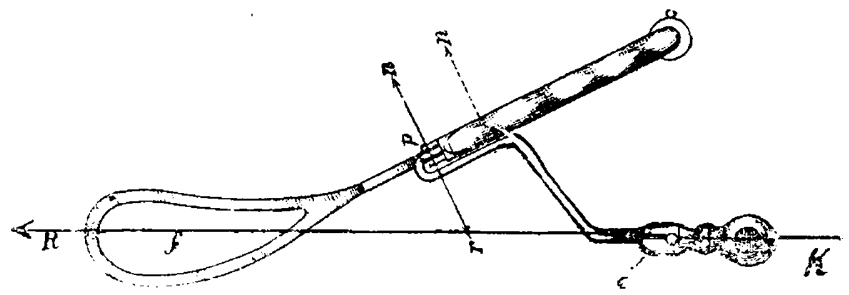

Fig. 9.-Side view of forceps, with its mechanical effect: KR. direction of resiatance as met with at the superior stritit f, center of blade; D, ball and socket joint. Notice that the socket piece and the ball end of the rod are in the samestraight line. P, decussation of shanks showing the socket receiving the hook end of the traction rod; pn, pe, the actual
expression of the force evolved from the lever effect of the traction-rod; expression of the force evolved from the lever
ca, the direct leverage force translated to pn.

of the instrument to the canal is possessed by no other non-rigid forceps. There is, in fact, no other instrument that will permit of rotation during the traction, and allow of axis-traction in every case.

Bearing in mind the correction of the conventional error hereinbefore mentioned, a very adequate descrip. tion of the forceps will be found in the Medical Record of June 10, 1893.

This instrument is manufactured by Richard Kny \& Co., of New York city.

\section{THE VAGINAL ROUTE FOR OPERATIONS ON} THE PELVIC VISCERA.

Read in the Section on Obstetrics and Diseases of Women at the Fortysixth Annual Meeting of the American Medical Association, at Baltimore, Md., May 7-10, 1895.

BY D. TOD GILLIAM, M.D. columus, oнio.

Everything indicates that we are about to have an epidemic of transvaginal surgery. Salpingectomy, cholecystotomy, nephrectomy and operations for appendicitis have ceased to be novelties and have therefore to an extent lost their charm. Men are merely boys grown up and, like boys, are fond of exploit. and adventure. This in the main results in good by advancing knowledge, developing manual dexterity and evolving new methods. . The pity is that in the name and guise of science and the legitimate pursuit of this noblest art so much is "done for self, to the detriment of the patient. The surgeon should never aspire to a record; the best interests of his patient, the advancement of his art and the greatest ultimate good to his race should be the aim and object of his life. To such an one, fame, honor and confidence of the profession and people will come in due time, and will come to stay. From this standpoint let us look into the merits and demerits of this new fad; endeavor to prescribe its scope and limits, and by comparison with older and better known methods strive to affix its proper status in gynecologic surgery.

That many operations on the pelvic viscera through the vagina are practicable is well known to every one at all conversant with gynecologic history, and there are few gynecologists of long or extensive practice who have not essayed most of them. Some years 
ago, indeed, it was the favorite and almost only method practiced, and there are doubtless elderly men in this assembly to-day who could give us pointers in vaginal surgery. We can all remember the time when abscesses and other fluid collections in the pelvis were attacked through the vaginal vault and when submucous and interstitial uterine fibroids, even of large size, were removed per vaginam. At a later period the uterine appendages and even ectopic gestation, ovarian cysts and the uterus itself were removed by this route. It remained for Péan and his collaborators to cap the climax by removing the fixed uterus by morcellation through the same channel. It is the latter, with its extensions to other conditions, that has given the wonderful impulse to transvaginal surgery that we witness to-day.

Let us briefly inquire into the advantages and disadvantages of the vaginal route, as compared with that by abdominal section, as it is between these that the issue lies. In the former we obviate section of the abdominal wall with its resultant scar. This scar is not only disfiguring and repulsive to the sensitive nature of the most sensitive of beings, but entails a feeling of weakness and insecurity; is at times painful and not infrequently the site of hernia. Furthermore, the omentum or bowel invariably becomes adherent to it and is liable to give rise to dis. tressing, if not dangerous sequelæ. It is now generally conceded that the shock of the vaginal operation as compared with that of abdominal section is trivial, because the intestines, the most sensitive of the abdominal viscera, are not exposed or handled. This is far-reaching, for not only may the shock itself be dangerous to life, but by diminishing vital resistance it predisposes to sepsis and cripples the functions of important organs. Intestinal paralysis does not occur.

Sepsis, the bane of abdominal surgery, is less frequent in the transvaginal surgery: 1 , because the pelvic peritoneum is better able to cope with germs; 2 , because the route of invasion is less direct and against the tide of outflowing serum; and 3 , because the germicidal powers of the splanchnic viscera are unabated. From a well.known fact that the vagina is the natural habitat of pathogenic germs of many varieties, that it is sometimes exceedingly foul, and from the supposed impossibility of render. ing it surgically clean, I, with many others, had a dread of entering the peritoneal cavity by that route and always, when possible, refrained from doing so. I now have no more hesitation on that score. I believe with douches aud a thorough scrubbing with creoline mollin we can render the vagina as aseptic as the skin. Again, in removing septic matters through the abdominal incision there is always a chance of peritoneal contamination and in many instances, as enucleating pus tubes, inundation of the cavity is unavoidable. This is always avoidable in the operation from below. Drainage through the vagina is incomparably more efficient than through the abdomen. The vaginal drainage is natural, perfect and with gravity. Abdominal drainage is against gravity. Not only so, but the animal fluids coating the tube or filling the meshes of the gauze afford an excellent culture medium for stray germs, which naturally make their way downward or inward. On the other hand, the outflow of fluids toward and into the va. gina, not only prevents the accumulation of septic matter in the pelvis, but washes away any that may be seeking entrance from below. So far the advantages are decidedly with the vaginal route, but it has its disadvantages. It is optional with the abdominal surgeon how much room he may make for himself. He can always keep the objective point under the eye or finger. He has ample facilities for seeing and knowing the position and surroundings, for avoiding important structures, ligating vessels and guarding against mischance. The ability to control circumstances and to advance with open eyes constitutes good surgery. Moreover his field is not abridged or limited. He can pursue the malady to the uttermost limits and he can preserve the healthy while eliminating the diseased. Should there be a mistaken diagnosis he can tack about and still be master of the situation. With the vaginal surgeon it is different. His field is limited; his operative space not amenable to optional extension. His facilities for meeting emergencies are meager. He is working in cramped quarters and ofttimes at a great disadvantage. It follows, then, that vaginal surgery has a sphere of its own. It can not aspire to supplant abdominal surgery nor yet in any large measure trench upon it. The possibilities of vaginal surgery will depend largely upon fortuitous circumstances. With a large, lax and shallow vagina many operative procedures may be feāsible, but with a narrow, deep and unyielding one, even the more common of the operations may be rendered difficult or impossible. I speak knowingly on this subject, for I have had experiences in both directions. I have removed submucous fibroids, pelvic. cysts and the malignant uterus as large as the fetal head, sometimes with comparative ease. I have signally failed to remove the fixed uterus on two occasions, on account of the narrowness, depth of the canal and unyielding structures about its outlet. In the first instance, incising the perineum failed to relieve the situation on account of the great depth of the canal. In the last, I was provided with Péan's retractors and all the appurtenances used by him. It was with the utmost difficulty that the retractors were introduced, and then, after prolonged effort it was found impossible to dilate the passage. As it is a cardinal rule never to go with the scissors or forceps where the eye or finger can not follow, I found it impracticable to complete the operation. Whether Péan or Jacobs could have done so, I know not, but I do know that the average surgeon would have failed as I did or done worse at a blind attempt at the impracticable. To my mind the field of transvaginal surgery includes hysterectomy of the normal or slightly enlarged uterus, the removal of the appendages when accessible, the drainage of the pelvic abscess, the morcellation of intra-uterine fibroids of moderate size and, possibly also, the extirpation of free cysts, low down in the pelvis.

It is unnecessary for me to say anything with reference to the technique, as this has been worked out so thoroughly as to leave little to be desired or expected. There are one or two points, however, to which I would like to call attention, and which I I have found in my own practice to have been of material advantage. A modified Trendelenburg position contributed greatly to the facilities for operating, by giving a much better light and by preventing the prolapse of intestines, which at times are annoying. Should there be purulent or septic collections in or about the field of operation this position would 
never be dispensed with. In many cases the short posterior retractor is vastly more efficient than the long ones used in Europe. It enables you to bring the vaginal vault within easy reach, and to follow the steps of the operation with much more ease and precision than when one has to work in the depths of a deep hole. Even in cases of fixation I have been able at times to use the short retractor to greater ad. vantage than the long one, and I am so imbued with its value that I would be loth to attempt any of the more serious operations through the vaginal vault without having one at hand. Feeling the want of a self-retaining retractor, that was simple and efficient, I devised the instrument which I now show you. You will observe that it consists of two pieces. The holder which passes under the sacrum and is held firm by the weight of the body and at the extremity of which is a steel pin for holding, and a screw for adjusting, the blade of the retractor at any angle. The retractor has a short handle with notches on the inner aspect and a ring at the end. In adjusting the speculum it is introduced as any other, then the index finger of the left hand is placed on the blade where it curves inward, while the thumb is placed under the free end or screw of the holder. Now, with the finger of the right hand in the ring of the handle, the retractor is guided into position through the slot on the side, while the blade and holder are approximated by pressure between the thumb and finger. Once in position it is easy to secure the desired degree of perineal retraction by increasing the pressure when it locks itself automatically. Now by turning the thumb screw the inclination of the blade may be changed at will. The mechanism is very simple, the movements are easy, the result all that could be desired. Besides the instrument, as a whole, takes up very littie space and can be carried almost as easily as a bivalve speculum.

\section{REMOTE RESULTS OF REMOVAL OF UTERINE APPENDAGES.}

Read in the Section on Obstetrics and Diseases of Women, at the Fortr sixth Aunual Meeting of the American Medical Association, at Baltimore, Md., May 7-10, 1895.

\section{BY E. E. MONTGOMERY, M.D.}

PHILADEIPHIA, PA.

The introduction of the operation for removal of the tubes and ovaries as advocated almost simultaneously by Battey, Tait and Hegar, though upon distinctly different lines, opened up an extensive field for the practice of abdominal surgery. The enthusiasm of their followers necessarily resulted in the practice of the procedure in many cases when it was of doubtful utility. Battey advocated the operation for intolerable dysmenorrhea; Tait for distinctly recognizable pathologic lesions in tubes and ovaries, principally of an inflammatory character; Hegar to produce an artificial menopause in cases of uterine hemorrhage otherwise uncontrollable.

These distinctly defined indications soon became extended, so that pelvic pain of slight degree, whether associated with menstruation or not, has been considered an excuse for the removal of the organs. Ovaries and tubes have been removed in which careful macroscopic investigation has failed to disclose much, if any, evidence of disease.

The experience of over twenty years has afforded opportunity to study the immediate and remote effects of operations which, at least, must have a profound effect upon the mental and physical nature of the individual in the interference with the procreative function.

It is a well recognized law of nature that unused faculties atrophy, so that it is not surprising to find that after castration of the unmarried female, the genital organs become smaller, the vaginal rugæ disappear and the mucous membrane is thinner and paler. In the married or those who subsequently marry, sexual desire becomes quiescent or even disappears, and to some the marital relation is painful and repugnant. Instead of being a willing and sympathetic partner in the act, she passes through the stages of indifference and passive suffering to disgust and distress.

If the condition just mentioned were the only unpleasant symptom, it would be a matter of but little moment. The most marked influence is upon the nervous system. It is generally recognized that the climacteric is a critical period in the life of every woman, one to which she looks forward with anxiety, realizing that hereditary traits and unpleasant phe. nomena are likely then to manifest themselves. If this be true when the woman matures and reaches the period undisturbed, how much more likely is she to suffer when the climacteric is abruptly and artificially induced, without nature having had an opportunity to prepare her forces for the changed relation.

Every operator is familiar with the flushing, vasomotor disturbances not unfrequently affecting the action of every organ of the body. These do not always disappear in a short period as in the natural menopause, but may continue for years, being a constant source of annoyance and discomfort. While it is true that many patients recover an appearance of good health who were previously great sufferers, in many this improvement will be found to be but tem. porary. Frequent attacks of neuralgia or neurasthenic pain in both ovarian regions as intense as prior to operation, in the uterus, in the intestines, the bladder, or the rectum; insomnia, restlessness and irritability of temper are not uncommon, Even if the individual may have had no desire for offepring, the assurance that motherhood is denied her frequently leads to intense longing for a child. When, however, she has been desirous for children and the possibility is precluded by an operation, the declining sexual appetite not unfrequently leads to a profound melancholy in which she feels life without solace or comfort.

Changes in pigmentation have been observed and it has been asserted that through meustruation certain material is eliminated which in its cessation is retained, producing splotches and discolorations of the skin, or the profound nervous manifestations.

The mental and nervous phenomena may be actuated or aggravated by the sequelæ of the surgical procedures, such as fistulæ, ventral herniæ, painful cicatrices, adhesions of intestines to the uterus, to the stumps, and to the bladder; cellular exudation, infection of ligatures, and the formation of abscess, immediately following the operation or years later.

A case recently came under my observation where, over four years after castration for bleeding fibroid, an abscess formed, which opened in the upper part of the cicatrix. An incision disclosed a large loop of silk in the abscess cavity.

Leucorrhea and irregular profuse uterine hemor. 